\title{
ON SUBADDITIVITY OF ANALYTIC CAPACITY FOR TWO CONTINUA
}

\author{
Dedicated to Professor Mitsuru Ozawa on his 60th birthday
}

By Nobuyuki Suita

1. Introduction. Let $K$ be a compact set in the complex plane $C$ and let $\Omega(K)$ be the connected component of $C-K$ containing the point at infinity. The analytic capacity $\gamma(K)$ of $K$ is defined by

$$
\gamma(K)=\sup _{\|f\| \leq 1}\left|f^{\prime}(\infty)\right| .
$$

Here $f(z)$ is a holomorphic function in $\Omega(K)$ whose expansion at the point at infinity is given by

$$
f(z)=f^{\prime}(\infty) / z+a_{2} / z^{2}+\cdots
$$

and $\|f\|$ denotes the supnorm of $f$.

The following problem was first raised by Vituškin [6]. For two disjoint compact sets $K_{1}$ and $K_{2}$, is there an absolute constant $M$ such that the following semiadditivity holds

$$
\gamma\left(K_{1} \cup K_{2}\right) \leqq M\left(\gamma\left(K_{1}\right)+\gamma\left(K_{2}\right)\right) ?
$$

We can find this problem and partial answers in many articles and books $[1,2,3,6,7]$. It was shown by Mel'nikov [3] that if $K_{1}$ and $K_{2}$ are separated by an analytic curve $c$, then there exists a constant $M(c)$ depending on $c$ for which (1) holds. This shows that the constant $M$ possibly depends on positions of two sets.

In the present paper we shall establish the following subadditive inequality

$$
\gamma\left(K_{1} \cup K_{2}\right) \leqq \gamma\left(K_{1}\right)+\gamma\left(K_{2}\right)
$$

for arbitrary disjoint continua $K_{1}$ and $K_{2}$. To prove (2) we shall solve an auxiliary extremal problem.

2. Extremal problem. Let $\Omega$ be a doubly connected domain containing the point at infinity. We suppose that its boundary components are continua $K_{1}$ and

Received March 22, 1983 
$K_{2}$. Let $\mathscr{T}$ be the family of univalent functions $\phi$ defined on $\Omega$ with a normalization

$$
\phi(z)=z+a_{1} / z+\cdots \quad \text { at } \infty .
$$

We denote by $\phi\left(K_{j}\right)$ the boundary component of the image domain $\phi(\Omega)$ under $\phi$ which corresponds to $K_{j}(j=1,2)$. Our problem is to minimize Cap $\phi\left(K_{1}\right)+\operatorname{Cap} \phi\left(K_{2}\right)$ within $\mathscr{F}$, Cap $\phi\left(K_{j}\right)$ being logarithmic capacity of $\phi\left(K_{j}\right)$. We prove

THEOREM1. The minimum of the quantity Cap $\phi\left(K_{1}\right)+$ Cap $\phi\left(K_{2}\right)$ is attained by the conformal mapping $\phi_{0}$ which maps $\Omega$ onto a parallel slit domain $\Delta$ whose boundary lies on a straight line.

Proof. Since capacity is invariant under rigid motions, we consider the same problem within a slightly larger class $\tilde{\mathcal{F}}$ of univalent functions $\phi$ whose expansion admits

$$
\phi(z)=e^{\imath \theta} z+a_{0}+a_{1} / z+\cdots \quad \text { at } \infty .
$$

There exists a unique radial slit disc mapping $\phi(z)$ in $\tilde{\mathcal{F}}$ such that $\psi\left(K_{1}\right)$ is a disc $|w| \leqq r$, say and $\phi\left(K_{2}\right)$ is a line segment $a \leqq w \leqq b(a>r)$. Then Cap $\phi\left(K_{1}\right)=r$ and we know that $r$ is equal to the minimum of Cap $\phi\left(K_{1}\right)$. For these facts see Sario and Oikawa ([5], pp. 148-158). By an elementary mapping $S_{r}(w)=$ $r(w / r+r / w), \phi\left(K_{1}\right)$ is mapped onto a line segment $[-2 r, 2 r]$ with the same capacity $r$. Then $\phi\left(K_{2}\right)$ is mapped onto a new segment $\left[a^{\prime}, b^{\prime}\right]$. We show that $\operatorname{Cap}\left[a^{\prime}, b^{\prime}\right]=\left(b^{\prime}-a^{\prime}\right) / 4$ is the minimum of $\operatorname{Cap} \phi\left(K_{2}\right)$ within $\tilde{T}$. In fact by a parallel displacement, $\left[a^{\prime}, b^{\prime}\right]$ is moved onto $\left[-2 r^{\prime}, 2 r^{\prime}\right], r^{\prime}=\left(b^{\prime}-a^{\prime}\right) / 4$. The conformal mapping $S_{r^{\prime}}^{-1}$ maps the last domain onto another radial slit disc, $K_{2}$ corresponding to the disc $|w| \leqq r^{\prime}$. Hence $r^{\prime}$ is the minimum of Cap $\phi\left(K_{2}\right)$. Thus $r+r^{\prime}$ is the desired minimum within $\tilde{\mathscr{F}} . S_{r^{\circ}} \phi$ is clearly an extremal function. It is easy to see that by composition of elementary mappings an extremal function $\phi_{0}$ can be constructed in $\mathscr{F}$ as stated in the theorem, which completes the proof.

Remark. The uniqueness of the extremal function in $\mathscr{F}$ is deduced from that of a radial slit disc mapping. Indeed the radial slit disc mapping $\psi$ with $\lim _{z \rightarrow \infty} \psi(z) / z=1$ is a unique extremal mapping minimizing radii within the family of univalent functions $\phi$ satisfying $\lim _{z \rightarrow \infty} \phi(z) / z=1$ and that $\phi\left(K_{1}\right)$ is a disc centered at the origin. It is not difficult to derive the uniqueness directly by means of the method of extremal metrics.

\section{Subadditivity. We prove}

THEOREM 2. If $K_{1}$ and $K_{2}$ are mutually disjoint continua, then the inequality (2) holds. 
Proof. If $\Omega\left(K_{\jmath}\right) \subset \Omega\left(K_{k}\right)(j \neq k)$, the inequality is trivial. Otherwise $\Omega\left(K_{1} \cup K_{2}\right)$ forms a doubly connected domain. For simplicity's sake we write $K_{1}, K_{2}$ for its boundary components which have the same analytic capacities as the original continua. Let $\phi_{0}$ be the extremal mapping for this domain in Theorem 1 . Pommerenke showed that for a compact set on a straight line its analytic capacity is equal to a quater of its total length [4]. Since the analytic capacity is invariant under a conformal mapping with the normalization (4), we have

$$
\gamma\left(K_{1} \cup K_{2}\right)=\gamma\left(\phi_{0}\left(K_{1}\right)\right)+\gamma\left(\phi_{0}\left(K_{2}\right)\right) .
$$

Since the analytic capacity is equal to the capacity for a continuum [7], we obtain the assertion from the minimum property in Theorem 1.

\section{REFERENCES}

[1] Brannan, D. A. And J.C. Clunie, Aspect of contemporary complex analysis, Academic Press (1980).

[2] Garnett, J., Analytic capacity and measure, Springer Lecture Notes in Math. 297 Springer-Verlag (1972).

[3] Mel'Nikov, M.S., Estimate of the Cauchy integral along an analytic curve, Math. Sbornik 71 (1966), 503-515 (Russian), Amer. Math. Soc. Translation ser. 2, 80 (1969), 243-256.

[4] Pommerenke, C., Über die analytische Kapatitat, Arch. der Math. 11 (1960), $270-277$.

[5] Sario, L. And K. Oikawa, Capacity functions, Springer-Verlag (1969).

[6] Vituśkin, A.G., The analytic capacity of sets in problems of approximation theory, Uspehi Mat. Nauk 22 (1967), 141-199 (Russian), Russian Math. surveys 22 (1967), 139-200.

[7] ZaLCMAN, L., Analytic capacity and rational approximation, Springer Lecture Notes in Math. 50, Springer-Verlag (1968).

Department of Mathenatics

Tokyo Institute of Techinology 